\title{
EDUCATION FOR RURAL SECTOR USING KNOWLEDGE NETWORK
}

\author{
B.G. Sangameshwara, U.M. Mallikarjuna Swamy" \\ Principal, S.J. College of Engg,, Mysore - 570 006, Karnataka, India. \\ bgsangam@yahoo.com \\ * Lecturer, Dept. of Information Science \& Engg., Mysore - 570 006, Karnataka, India. \\ mallisjce@yahoo.com
}

\begin{abstract}
Rural development in the Indian / III world context is still dominated by rural way of life up to now and likely to be for quite some time in the future. By simple statistics, up to $80 \%$ of the population lives in villages, or semi urban environment. Thus rural development / III world development has much more to do with attitudes, perceptions and sensitivities, and less with technologyper-se. The education for rural people using information technology (IT) in nural areas is very much required for the present scenario. If the people in the rural areas are educated, we can expect tremendous economy growth in the developing countries like India, China etc. and the African Continent. The main aim is to give IT based training and education for the rural peoples in the various part of Karnataka state, India using Knowledge Network.
\end{abstract}

Key words: IT, Education, Knowledge Network.

\section{INTRODUCTION}

Ever since its inception Information Technology (IT) has expanded its influence over all domains. IT has now become an integral part of all facets of business, education, research etc. Unfortunately, IT has not made much penetration into the rural sector. Hence, the developmental activities in the rural arena especially in Asia and Africa have not witnessed much of 
acceleration in its growth. Information Technology is the essential tool for economic development and material well-being in our age; it conditions power knowledge and creativity; it is for the time being, unevenly distributed within countries and between countries; and it requires, for the full realization of its development value, an inter-related system of flexible organizations and information-oriented institutions [1,2].

Our work proposes a novel approach to overcome the short comings in the rural development. IT is a key factor to alleviate the rural sector and can be leveraged to impart information, knowledge and education to the rural populace. It envisages a collaborative role to be played between Urban Nodes that can be any information-oriented intuition or any organization and Rural Nodes that is the recipient of services.

In computing, a Content Management System (CMS) is a system used to organize and facilitate collaborative creation of documents and other content. A CMS is frequently a web application used for managing websites and web content, though in many cases, Content Management Systems requires special client software for editing and constructing articles [3]. Plone is a content management framework that works hand-in-hand and sits on top of 'Z' Object Publishing Environment (Zope), a widely-used Open Source web application server and development system [4]. Plone powered websites offer dynamically updating the data and authorizes many users to update information based on their membership. This ensures a large dissemination of updated information.

\section{LEVERAGING IT FOR RURAL EDUCATION}

Unlike the urban students, the rural students have to mostly compromise with the quality of instructors. Thus they are bereft of the experience of being taught by domain experts or at least by those who are acquainted with the domain. Our work envisages a modest infrastructure in the education centers in the rural areas like availability of computers and internet facilities to solve this issue.

Our work involves a close collaboration between centers of excellence in education, knowledge institutes, industry that we hence forth address as Knowledge Centers situated in the urban areas and the rural education centers. Thus, knowledge can be imparted from any of the collaborative partners and the rural education center being the ultimate consumer of service. Students in the rural education center can extensively use the internet to acquire domain specific information. Since, the information on 
the internet is unevenly spread across and cannot be uploaded from a single mount point we propose to develop a web portals that can be collaborative in nature. Such collaboration is possible by employing CMS.

The Knowledge Centers can upload the information beneficial to the students from their place dynamically and this information can range from simple text file to advanced multi-media files. Since, we envisage a single mount point the students can procure knowledge at a single place on the web as well as post their queries onto the same point.

We christen such an arrangement as 'Knowledge Network'. Our work focuses on three major traits of the Knowledge Network.

- Flexibility: This feature emphasizes the need for the uploading/downloading of the information dynamically from any part of the world without pushing the onus of document publication on the webmaster. This would ensure the speedy dissemination of information and would ensure closer interaction between the students and the domain experts.

- Reliability: This feature emphasizes the fact that the whole arrangement must be reliable such that all the actors of the Knowledge Network can perform their task without any hindrance such as server crash, network failure etc. Hence, the Knowledge Network must be equipped with features to cope up with crisis like server crash and also the safe storage of all the data.

- Security: Since the information can be uploaded from different points by different set of people the authenticity of the information and the credibility of the information providers is an important issue. Hence the CMS must contain a secure work flow and membership feature to enable only authentic persons to have the authority to manage critical access and privilege.

\section{DESIGN OF KNOWLEDGE NETWORK}

The proposed Knowledge Network is defined to ensure the larger dissemination of information among the rural populace and this flow of information must be in such a manner that the system must extend flexibility and reliability. In order to offer flexibility a Content Management System like Plone is considered in the Knowledge Network. This will ensure the 
dynamic update of data online and this update can be performed from any where in the World Wide Web (WWW). The reliability of the network and the data fetching is ensured by employing the Cluster on Demand Architecture (Coda) and setting up replicated servers using Coda.

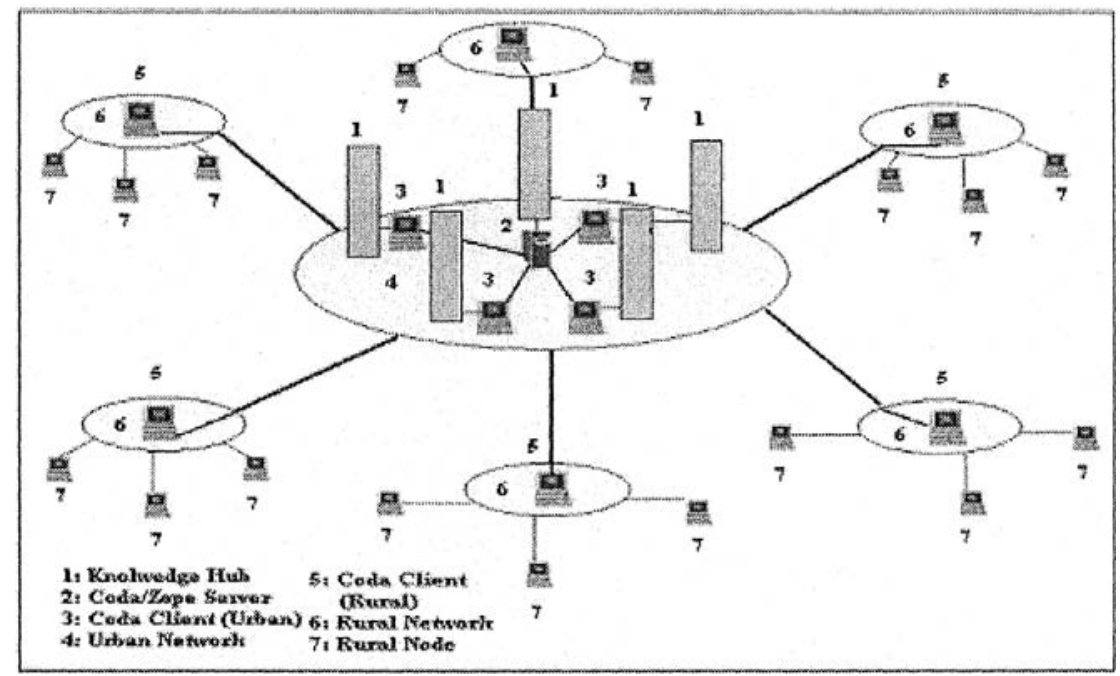

Figure 1. Design of the proposed Knowledge Network

The Knowledge Network consists of seven major components.

(1) Knowledge Institutes: The Knowledge Institutes have a crucial role in the Knowledge Network. The Knowledge Institutes host the Coda Server/Clients and the Zope Application Server. The Knowledge Institutes consist of domain experts who can share knowledge about IT and other creative issues with the rural populace leveraging the technology and the high speed networks. Since, Plone CMS is implemented the domain experts/knowledge leaders can feed information on the website which can viewed through the Rural Node over the Rural Network. There can be many such Knowledge Institutes than can play a collaborative role by dynamically authoring the WebPages.

(2) Coda/Zope Server: Coda is a state-of-the-art experimental file system [5]. The Coda Server contains one-to-one partitions for its clients. This partition on the server is visible to its clients in a virtualized manner. In the above case the Coda Server is integrated with Zope Web Application Server and the Zope Object Database (ZODB). Any changes made in the website or any updates are recorded in the ZODB. There can be any number of Coda 
Servers and each of them undergo perfect replication of data and in the above case each server may have a copy of ZODB and any updates are reflected on the ZODB copy of each server.

(3) Coda Client: The Coda Client in the above case serves as the web replication server. The Coda Client is connected to the Coda Server and each client owns an individual partition on the Coda Server. The data in the partition on the server is visible to the client, though it is not stored on the client. The Coda Client has caching and disconnected operation features which enable it to store the important files in its local disk cache. This provision enables the client to store the ZODB available on the server partition into its local cache. Thus, in case if the connection between the client and the server fails due to some network failure the client will be still containing the important data and in this case the updated copy of ZODB

(4) Urban Network: The Urban Network is the supplier of knowledge and services to the Rural Network. The Urban Network must consist of high speed network lines and can be heterogeneous in nature. The Urban Network hosts the Coda Server, Coda Clients (Replication Servers), Knowledge Institutes.

(5) Coda Client (Rural): This Coda Client will also contain a copy of the ZODB and hence serve as Replication Server. Each rural network can contain a Coda Client. This will ensure a quick access to the server. The Coda Client can be maintained by a nodal center in each rural network. These nodal centers can serve as the knowledge acquisition points where rural people can provide useful information legible to be uploaded on the website.

(6) Rural Network: The Rural Network is the recipient of knowledge and services from the Urban Network. The Rural Network can be connected to the Urban Network through Optical Backplane Engineering techniques which would ensure a reliable and fast data transfer. The rural network consists of nodes which can systems in educational places like schools or composite colleges etc. The Rural Network can also consist of Coda Client (Replication Servers).

(7) Rural Node: The Rural Node is basically an end system which is a part of the rural network. The Rural Node is the end recipient of knowledge and services. The Rural Node is connected to the Coda Server/Replication Server through high speed network lines. Information flow is presented to the user 
through Plone CMS which runs on the Coda Server/Replication Server. Since Plone CMS allows the dynamic updating of data on the website, the user can make data update from the rural node also. In an interactive learning session there can exist feedbacks to the instructor from the students. Since, a CMS is considered such feedbacks can be posted through a single login.

\section{CONCLUSION AND FUTURE WORK}

The Knowledge Network is developed with the objective of providing quality distance education to the rural students and upgrading them to a degree of knowledge comparable to their urban peers. The penetration of IT into the rural world will result in the accelerated growth of the villages and will establish a balance between the rural populace and their urban peers. The rural traders will benefit from the knowledge network as they can procure the up-to-date prices of the commodities and can bid for fair prices. The agriculturists will be exposed to a knowledge arena and will introduce them to the urban markets and will result in exponential profits due to the absence of middle men in the dealings.

The Knowledge Network proposed relies heavily on Plone CMS and Coda. Plone is an open source project. Since, this network is envisaged for developing countries the Plone Community must direct sincere efforts towards internationalization. This will result in the Plone web pages being developed in different languages. There exists a project within the Plone Community called ' $\mathrm{i} 18 \mathrm{n}$ ' which aims at delivering Plone CMS in various languages. This feature will be beneficial for developing worlds like Asia and Africa where IT has not made much of penetration in the rural sector. Projects like 'il8n' will foster imparting education in the regional languages leveraging IT.

\section{REFERENCES}

1. Maneul Castells, Information Technolgy, Globalization and Social Development (UNRISD Discussion paper No. 114, September 1999).

2. B.G. Sangameshwara, Ph.D. Thesis "Information Technology for Rural Development: A System's Approach", University of Mysore, India.

3. Wikipedia, http://en.wikipedia.org/wiki/Content management system

4. Andy Mckay, The Definitive Guide to Plone (2004).

5. Peter J. Braam, Linux Journal (June 1998). 